\title{
Influence of velocity dispersions on star-formation activities in galaxies ${ }^{\star}$
}

\author{
Tsan-Ming Wang (王贊銘 $)^{1,2,3}$ and Chorng-Yuan Hwang (黃崇源) ${ }^{3}$ \\ 1 Max-Planck-Institut für Radioastronomie, Auf dem Hügel 69, 53121 Bonn, Germany \\ 2 Argelander-Institut für Astronomie, Universität Bonn, Auf dem Hügel 71, 53121 Bonn, Germany \\ e-mail: twan@uni-bonn.de \\ 3 Graduate Institute of Astronomy, National Central University, No. 300, Zhongda Rd., Zhongli Dist., Taoyuan City 320, Taiwan
}

Received 15 February 2020 / Accepted 15 June 2020

\begin{abstract}
We investigated the influence of the random velocity of molecular gas on star-formation activities of six nearby galaxies. The physical properties of a molecular cloud, such as temperature and density, influence star-formation activities in the cloud. Additionally, local and turbulent motions of molecules in a cloud may exert substantial pressure on gravitational collapse and thus prevent or reduce star formation in the cloud. However, the influence of gas motion on star-formation activities remains poorly understood. We used data from the Atacama Large Millimeter/submillimeter Array to obtain ${ }^{12} \mathrm{CO}(J=1-0)$ flux and velocity dispersion. We then combined these data with 3.6 and 8 micron midinfrared data from the Spitzer Space Telescope to evaluate the effects of gas motion on starformation activities in several nearby galaxies. We discovered that relatively high velocity dispersion in molecular clouds corresponds with relatively low star-formation activity. Considering the velocity dispersion as an additional parameter, we derived a modified Kennicutt-Schmidt law with a gas surface density power index of 0.84 and velocity dispersion power index of -0.61 .
\end{abstract}

Key words. galaxies: spiral - galaxies: star formation - galaxies: ISM

\section{Introduction}

The history of the evolution of galaxies is one of the most critical points of inquiry in modern astronomy. Stars are a major component of galaxies, and they should be examined in investigations of galaxies' pasts as well as their potential courses of development (e.g., McKee \& Ostriker 2007). However, the detailed mechanism by which gas turns into stars remains a mystery (e.g., Federrath et al. 2016).

Self-gravitational collapse is fundamental to the process of star formation in a molecular cloud. However, the star-formation rate (SFR) in a galaxy may be considerably higher than the observed values if the star-formation activities only result from gas free-fall in a cloud (e.g., Shu et al. 1987; Padoan et al. 2014). Molecular clouds' physical properties, such as turbulence and magnetic field, are believed to influence the capacity for star formation (e.g., Kirk et al. 2009). Magnetic fields play a role in star formation (e.g., Hull et al. 2017; Higuchi et al. 2018). Zhang et al. (2014a) present SMA observations of 14 massive molecular clumps in the $345-\mathrm{GHz}$ band and conclude that magnetic fields at the core scale of $0.01-0.1 \mathrm{pc}$ tend to be relatively organized; furthermore, the magnetic fields of over $60 \%$ of the cores are perpendicular to the major axes of the cores. These results demonstrate that magnetic fields play a critical role in the collapse and formation of dense cores. Additionally, gas turbulence in the interstellar medium (ISM) is believed to regulate starformation activities (e.g., Norman \& Silk 1980; Federrath 2013). For example, Orkisz et al. (2017) used ${ }^{13} \mathrm{CO}(J=1-0)$ to study the turbulence in the Orion B molecular cloud. The researchers

\footnotetext{
* The reduced datacubes are only available at the CDS via anonymous ftp to cdsarc.u-strasbg.fr (130.79.128.5) or via http://cdsarc.u-strasbg.fr/viz-bin/cat/J/A+A/641/A24
}

discovered that the motions in the cloud are mostly solenoidal. This finding is consistent with the cloud's low SFR.

Larger-scale SFR surface densities have been reported as proportional to gas surface density with a positive power-law index $\mathrm{N}$ and a coefficient A (Schmidt 1959):

$\Sigma_{\mathrm{SFR}}=A\left(\Sigma_{\mathrm{gas}}\right)^{N}$.

Following this equation and conception, Kennicutt (1998) derived an empirical law (the Kennicutt-Schmidt, KS, law) regarding the relationship between SFR surface densities and gas surface densities. In this law, Kennicutt used observational data from disk and starburst galaxies:

$\Sigma_{\mathrm{SFR}}=(2.5 \pm 0.7) \times 10^{-4}\left(\frac{\Sigma_{\mathrm{gas}}}{1 M_{\odot} \mathrm{pc}^{-2}}\right)^{1.45 \pm 0.15} M_{\odot} \mathrm{yr}^{-1} \mathrm{kpc}^{-2}$.

Researchers agree that additional parameters should be established to enable a more accurate description of the SFR. Green et al. (2010) identified a positive correlation between velocity dispersion and the SFR using the $\mathrm{H} \alpha$ emission line in kiloparsec scales. They discovered that the correlation is independent of redshift when the $\mathrm{H} \alpha$ luminosity is greater than $10^{42} \mathrm{erg} \mathrm{s}^{-1}$.

The aforementioned studies have provided information regarding star formation within a single molecular cloud or within a whole galaxy. However, it is still hard to establish a unified model that connects star-formation activities on these two different scales. Moreover, there are different SFR tracers with different calibrations, such as $\mathrm{H} \alpha+24 \mu \mathrm{m}$, far-ultraviolet $+24 \mu \mathrm{m}$, and far-ultraviolet $+22 \mu \mathrm{m}$ (e.g., Hao et al. 2011; 
Cortese 2012; Casasola et al. 2017). These star-formation tracers have been applied to study the star-formation law in different scales. Eales et al. (2010) used $\mathrm{H} \alpha+24 \mu \mathrm{m}$ as the SFR tracer and found KS law power indices of 1.29 for M 99 and 1.67 for M 100 with a resolution $\sim 2 \mathrm{kpc}$. Kennicutt et al. (2007) used $\mathrm{H} \alpha+24 \mu \mathrm{m}$ as the SFR tracer and reported that the KS law is valid within NGC 5194, but that the power-law index varies from 1.37 to 1.56 when the resolution decreases from 2 to $0.5 \mathrm{kpc}$. Leroy et al. (2008) used far-ultraviolet $+24 \mu \mathrm{m}$ as the SFR tracer to explore the star-formation law within nearby galaxies with a spatial resolution of $800 \mathrm{pc}$; they found that the star formation efficiency of $\mathrm{H}_{2}$ is about $5.25 \times 10^{-10} \mathrm{yr}^{-1}$. Bigiel et al. (2008) used far-ultraviolet $+24 \mu \mathrm{m}$ as the SFR tracer and found a KS law power index of about 1 with a spatial resolution of $750 \mathrm{pc}$. Momose et al. (2013) used $\mathrm{H} \alpha+24 \mu \mathrm{m}$ as the SFR tracer to study the KS law within ten nearby spiral galaxies; they revealed KS law power indices of 1.3 for $750 \mathrm{pc}$ resolution and 1.8 for $500 \mathrm{pc}$ resolution. Pereira-Santaella et al. (2016) used $\mathrm{Pa} \alpha$ as the SFR tracer and discovered that the correlation between $\Sigma_{\text {SFR }}$ and $\Sigma_{\mathrm{H}_{2}}$ is weak, and that the star-formation law loses applicability at the scale of $250 \mathrm{pc}$ in IC 4687 . Ford et al. (2013) used farultraviolet $+24 \mu \mathrm{m}$ as the SFR tracer to investigate the $140 \mathrm{pc}$ resolved star-formation law within M 31; they show KS law power indices of 2.03 between the $\Sigma_{\text {gas }}-\Sigma_{\mathrm{SFR}}$ relation, and 0.60 between the $\Sigma_{\mathrm{H}_{2}}-\Sigma_{\mathrm{SFR}}$ relation. Miura et al. (2014) used farultraviolet $+24 \mu \mathrm{m}$ as the SFR tracer to study the $100 \mathrm{pc}$ resolved star-formation law within M 33; they obtained a KS law power index of 1.04 for the $I_{\mathrm{CO}(3-2)}-\Sigma_{\mathrm{SFR}}$ relation. These varying starformation laws suggest that the global-scale Schmidt law cannot sufficiently explain the SFR on hundred-parsec scales, and other parameters might be needed to describe a more universal hundred-parsec star-formation law.

The goal of the present study is to evaluate the influence of gas motion on SFR within a scale of approximately $200 \mathrm{pc}$ in nearby galaxies. The present study offers a bridge between local and global star-formation models. In Sect. 2, we introduce selected data and describe their properties. In Sect. 3, we present our results and derive a modified KS law. Finally, in Sect. 4, we summarize our results and present conclusions.

\section{Methods}

In this section, we present our method for determining molecular gas density, velocity dispersion, and SFR. Subsequently, data are listed, and related properties are discussed.

We investigated the influence of gas motion on SFR within an area of approximately $200 \times 200 \mathrm{pc}^{2}$. We obtained ${ }^{12} \mathrm{CO}(J=$ 1-0) data from the Atacama Large Millimeter/submillimeter Array (ALMA) archive website for six nearby galaxies, each of which is at a luminosity distance less than 17.5 Mpc. Details regarding the selected galaxies and the ALMA array configurations are listed in Table 1 . We performed data calibration and imaging using the Common Astronomy Software Applications (CASA) package following the guidelines of the ALMA Cookbook (Ott \& Kern 2016). We calibrated the raw data by using the scriptForPI.py scripts provided by the Joint ALMA Observatory. Calibrated visibilities were then imaged with the CASA version 4.6.0 with robust weighting. The results reveal the synthesized beams with major axes of approximately $2^{\prime \prime}-3^{\prime \prime}$ and velocity resolutions of approximately $2-5 \mathrm{~km} \mathrm{~s}^{-1}$ (Table 2).

Figure 1 illustrates the box distribution of NGC 1808; the box distributions of other galaxies are presented in the Appendix. Details regarding the box sizes in various galaxies are listed in Table 2. To test molecular properties at a similar physical scale, we estimated velocity dispersions and the ${ }^{12} \mathrm{CO}(J=1-0)$ flux inside selected boxes with sizes of approximately $200 \times 200 \mathrm{pc}^{2}$ along arms or bars in the galaxies. We obtained the velocity dispersion from the Gaussian fits of the spectra in the selected starformation regions as illustrated in Fig. 2. For the star-formation regions with multiple components in the spectra as shown in the bottom of Fig. 2, we fitted the spectra with multiple Gaussian fits and estimated the velocity dispersion $\sigma$ of the spectra using the peak intensity weighted dispersions derived from the multiple Gaussian fits:

$\sigma=\frac{\sum_{i} I_{i} \sigma_{i}}{\sum_{i} I_{i}}$

where $I_{i}$ is the peak intensity and $\sigma_{i}$ is the dispersion of each Gaussian fit.

Several of our selected galaxies are classified as active galactic nuclei (AGNs): NGC 1808 (e.g., Ramos Almeida et al. 2009; Busch et al. 2017), NGC 1365 (e.g., Peretz \& Behar 2018; Venturi et al. 2018), NGC 3627 (e.g., Ho et al. 1997; VéronCetty \& Véron 2010), and the Circinus galaxy (e.g., Moorwood et al. 1996; Ramos Almeida et al. 2009). To avoid AGN-related effects, we did not select the data near the central regions of these galaxies.

We used Spitzer archive data to estimate the SFRs inside the same selected boxes of ${ }^{12} \mathrm{CO}(J=1-0)$ images. Because the pixel sizes of the ALMA and Spitzer images were different, the results regarding box positions and sizes could vary slightly between the calculations based on these two data sets. However, the differences were less than 5\%, and thus should not have affected the validity of the comparison between the ALMA and Spitzer data. The SFRs were estimated using $8 \mu \mathrm{m}$ (dust) luminosity following the approach proposed by Wu et al. (2005):

$\frac{\mathrm{SFR}}{\left(M_{\odot} \mathrm{yr}^{-1}\right)}=\frac{v L_{v}[8 \mu \mathrm{m}(\text { dust })]}{1.57 \times 10^{9} L_{\odot}}$.

The $8 \mu \mathrm{m}$ (dust) emission is an ideal tracer for small-scale star formation because the $8 \mu \mathrm{m}$ (dust) emission has a high spatial resolution compared to the longer wavelength mid-infrared emissions, such as 22 or $24 \mu \mathrm{m}$. Besides, the $8 \mu \mathrm{m}$ (dust) emission has less extinction and contamination compared to $\mathrm{H} \alpha$ emission. However, the $8 \mu \mathrm{m}$ (dust) emission might not be linearly proportional to the SFR at conditions with very intense or very weak star-formation activities (Wu et al. 2005). The disadvantage of SFR derived from the $8 \mu \mathrm{m}$ (dust) emission should have little influence on our results because our sample does not contain very intense star-formation regions. The $8 \mu \mathrm{m}$ (dust) luminosity was derived from the 3.6 and $8 \mu \mathrm{m}$ fluxes of the Spitzer data:

$f_{8 \mu \mathrm{m}}($ dust $)=f_{8 \mu \mathrm{m}}-\eta f_{3.6 \mu \mathrm{m}}$,

where $\eta$ is 0.232 (Leitherer et al. 1999; Helou et al. 2004).

\section{Results}

We investigated the relation between ${ }^{12} \mathrm{CO}(J=1-0)$ luminosity surface density, velocity dispersion, and SFR surface density for each galaxy. Figure 1 denotes the results for NGC 1808 as an example. We found that the ${ }^{12} \mathrm{CO}(J=1-0)$ luminosity surface density is highly correlated with the SFR surface density for most of the selected galaxy regions except for the bar region of NGC 3627 and the arm region of NGC 1365, the latter of which has only 11 data points. (Table 3 ). We also 
Table 1. Galaxy information.

\begin{tabular}{|c|c|c|c|c|c|}
\hline $\begin{array}{l}\text { Project code } \\
\text { (1) }\end{array}$ & $\begin{array}{l}\text { Galaxy } \\
\text { (2) }\end{array}$ & $\begin{array}{c}\text { Morphology }{ }^{(a)} \\
\text { (3) }\end{array}$ & $\begin{array}{c}\text { Inclination angle } \\
\text { (Degree) } \\
(4)\end{array}$ & $\begin{array}{l}\text { Distance }^{(a)} \\
(\mathrm{Mpc}) \\
(5)\end{array}$ & $\begin{array}{c}\text { ALMA array type } \\
\text { (6) }\end{array}$ \\
\hline 2013.1.00532.S & NGC628 & $\mathrm{SA}(\mathrm{s}) \mathrm{c}$ & $6^{(b)}$ & 10.2 & $12 \mathrm{~m}+\mathrm{ACA}+\mathrm{TP}$ \\
\hline 2012.1.01004.S & NGC1808 & (R)SAB(s) & $57^{(c)}$ & 9.08 & $12 \mathrm{~m}+\mathrm{ACA}+\mathrm{TP}$ \\
\hline 2012.1.00759.S & NGC4568 & $\mathrm{SA}(\mathrm{rs}) \mathrm{bc}$ & $66^{(d)}$ & 17.3 & $12 \mathrm{~m}$ \\
\hline 2013.1.00247.S & Circinus galaxy & $\mathrm{SA}(\mathrm{s}) \mathrm{b}$ & $40^{(e)}$ & 4.21 & $12 \mathrm{~m}+\mathrm{TP}$ \\
\hline 2015.1.01538.S & NGC3627 & $\mathrm{SAB}(\mathrm{s}) \mathrm{b}$ & $65^{(f)}$ & 9.04 & $12 \mathrm{~m}$ \\
\hline 2015.1.01135.S & NGC1365 & $\mathrm{SB}(\mathrm{s}) \mathrm{b}$ & $46^{(g)}$ & 17.5 & $12 \mathrm{~m}$ \\
\hline
\end{tabular}

Notes. This table presents (1) ALMA project code, (2) name, (3) identified morphology, (4) inclination angle, (5) luminosity distance toward us, (6) array configuration.

References. ${ }^{\left({ }^{\prime}\right)}$ The informations are obtained from NED. ${ }^{(b)}$ Shostak \& van der Kruit (1984). ${ }^{(c)}$ Reif et al. (1982). ${ }^{(d)}$ Vollmer et al. (2013). ${ }^{(e)}$ Chemin et al. (2003). ${ }^{(f)}$ Elmouttie et al. (1998). ${ }^{(g)}$ Ondrechen \& van der Hulst (1989).

Table 2. Data information and selected box size.

\begin{tabular}{lccccc}
\hline \hline $\begin{array}{l}\text { Galaxy } \\
(1)\end{array}$ & $\begin{array}{c}\text { Velocity resolution } \\
(2)\end{array}$ & $\begin{array}{c}\text { Box numbers } \\
(3)\end{array}$ & $\begin{array}{c}\text { Restoring beam size } \\
(4)\end{array}$ & $\begin{array}{c}\text { ALMA box size } \\
(5)\end{array}$ & $\begin{array}{c}\text { Spitzer box size } \\
(6)\end{array}$ \\
\hline NGC628 & $5 \mathrm{~km} \mathrm{~s}^{-1}$ & 97 & $2^{\prime \prime} .89 \times 1^{\prime \prime} .79$ & $4^{\prime \prime} .2 \times 4^{\prime \prime} .2(208 \mathrm{pc})^{2}$ & $4^{\prime \prime} \times 4^{\prime \prime}(198 \mathrm{pc})^{2}$ \\
NGC1808 & $2.5 \mathrm{~km} \mathrm{~s}^{-1}$ & 20 & $2^{\prime \prime} .27 \times 1^{\prime \prime} .30$ & $4^{\prime \prime} .25 \times 4^{\prime \prime} .25(187 \mathrm{pc})^{2}$ & $4^{\prime \prime} .2 \times 4^{\prime \prime} .2(185 \mathrm{pc})^{2}$ \\
NGC4568 & $5 \mathrm{~km} \mathrm{~s}^{-1}$ & 48 & $2^{\prime \prime} \times 2^{\prime \prime}$ & $2^{\prime \prime} .5 \times 2^{\prime \prime} .5(206 \mathrm{pc})^{2}$ & $2^{\prime \prime} .4 \times 2^{\prime \prime} .4(198 \mathrm{pc})^{2}$ \\
Circinus galaxy & $3 \mathrm{~km} \mathrm{~s}^{-1}$ & 11 & $2^{\prime \prime} .58 \times 2^{\prime \prime} .01$ & $11^{\prime \prime} \times 11^{\prime \prime}(206 \mathrm{pc})^{2}$ & $10^{\prime \prime} .8 \times 10^{\prime \prime} .8(202 \mathrm{pc})^{2}$ \\
NGC3627 & $3 \mathrm{~km} \mathrm{~s}^{-1}$ & $35+9^{(a)}$ & $3^{\prime \prime} .11 \times 2^{\prime \prime} .14$ & $4^{\prime \prime} .8 \times 4^{\prime \prime} .8(210 \mathrm{pc})^{2}$ & $4^{\prime \prime} .8 \times 4^{\prime \prime} .8(210 \mathrm{pc})^{2}$ \\
NGC1365 & $5 \mathrm{~km} \mathrm{~s}^{-1}$ & $11+26^{(b)}$ & $2^{\prime \prime} .36 \times 2^{\prime \prime} .06$ & $2^{\prime \prime} .4 \times 2^{\prime \prime} .4(204 \mathrm{pc})^{2}$ & $2^{\prime \prime} .4 \times 2^{\prime \prime} .4(204 \mathrm{pc})^{2}$ \\
\hline
\end{tabular}

Notes. This table presents (1) name, (2) velocity resolution of ALMA image cube, (3) number of boxes, (4) synthsized beam size, (5) size of ALMA box, (6) size of Spitzer box. ${ }^{(a)}$ We separated NGC 3627 into arm region (35 boxes) and bar region (9 boxes). ${ }^{(b)}$ We separated NGC 1365 into arm region (11 boxes) and bar region ( 26 boxes).

found positive correlations between the velocity dispersion and ${ }^{12} \mathrm{CO}(J=1-0)$ luminosity surface density for the selected galaxy regions, although the correlations are not significant for most of the selected galaxy regions (Table 4). Positive correlations between the velocity dispersion and SFR surface density are also identified for some of the galaxies (Table 5).

The relations between the ${ }^{12} \mathrm{CO}(J=1-0)$ luminosity surface density and SFR surface density for all the studied galaxies are presented in Fig. 3. We used the following formula and the SciPy orthogonal distance regression fitting package (Boggs \& Rogers 1990) to fit a linear relation for the log values between the $12 \mathrm{CO}(J=1-0)$ luminosity surface density and SFR surface density:

$$
\begin{aligned}
& \log \text { (SFR surface density })= \\
& \quad \alpha_{1} \log \left(L_{12} \mathrm{CO}(J=1-0) \text { surface density }\right)+C_{1} .
\end{aligned}
$$

The results of these fittings are denoted in Table 3. The galaxies NGC 3627 and NGC 1365 show clear bars in their optical images; we thus separated the data of NGC 3627 and NGC 1365 into the arm region and bar region of the galaxies. Most of the galaxies exhibited a positive correlation between the ${ }^{12} \mathrm{CO}(J=$ 1-0) luminosity surface density and SFR surface density except for the bar region of NGC 3627 and the arm region of NGC 1365. For some of the galaxies, a power-law index value close to the value of the traditional $\mathrm{KS}$ law was observed. We note that NGC 4568 and NGC 3627 have power-law index values much lower than that of the typical KS law. By contrast, NGC 1365 exhibited much higher power-law index values than that of the typical KS law. Table 3 indicates the results of Pearson's and Spearman's tests for the correlation between the ${ }^{12} \mathrm{CO}(J=1-0)$ luminosity surface density and SFR surface density. Except for the results for the arm region of NGC 1365 and the bar region of NGC 3627, most of the galaxies exhibited significant correlations in the results for the aforementioned variables.

The relations between the velocity dispersion and ${ }^{12} \mathrm{CO}(J=$ 1-0) luminosity surface density for all of the studied galaxies are denoted in Fig. 4. Most of the galaxies are characterized by a positive correlation between the velocity dispersion and ${ }^{12} \mathrm{CO}(J=1-0)$ luminosity surface density. We used the following formula and the orthogonal distance regression fitting package to fit a linear relation for the log values between the velocity dispersion and ${ }^{12} \mathrm{CO}(J=1-0)$ luminosity surface density:

$$
\begin{aligned}
\log \left(L_{12} \operatorname{CO}(J=1-0)\right. & \text { surface density })= \\
& \beta_{1} \log (\text { velocity dispersion })+C_{2} .
\end{aligned}
$$

The results of these fittings are indicated in Table 4. Table 4 presents the results of Pearson's test and Spearman's test for the positive correlations between the velocity dispersion and ${ }^{12} \mathrm{CO}(J=1-0)$ luminosity surface density. Most of the galaxies exhibited a significant positive correlation, except for the Circinus galaxy, NGC 628, NGC 1365, and the bar region of NGC 3627.

The results regarding the relations between the velocity dispersion and SFR surface density for all of the studied galaxies are presented in Fig. 5. We used the following formula and the orthogonal distance regression fitting package to fit a linear relation for the log values between the velocity dispersion and SFR surface density:

$\log ($ SFR surface density $)=\beta_{2} \log ($ velocity dispersion $)+C_{3}$. $(8)$ 

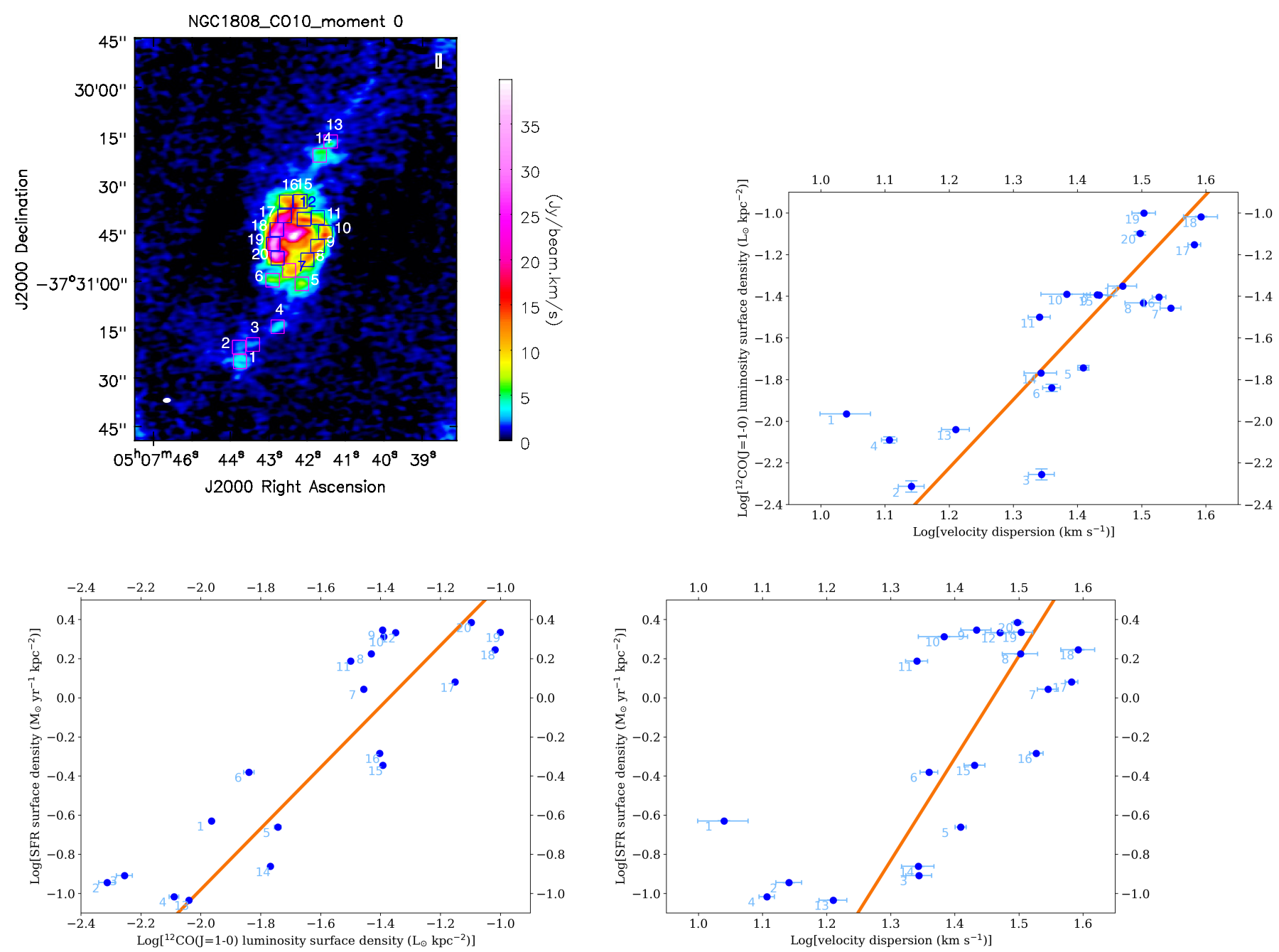

Fig. 1. Using NGC 1808 as an example in our data analysis. Top left: ${ }^{12} \mathrm{CO}(J=1-0)$ moment 0 map of NGC 1808. Top right: relation of the velocity dispersion and ${ }^{12} \mathrm{CO}(J=1-0)$ luminosity surface density of NGC 1808 . Bottom left: relation of the ${ }^{12} \mathrm{CO}(J=1-0)$ luminosity surface density and SFR surface density of NGC 1808. Bottom right: relation of the velocity dispersion and SFR surface density of NGC 1808 . Different star-formation regions are marked in different numbers. The orthogonal distance regression fittings for different relations are displayed in orange lines.

The results of these fittings are denoted in Table 5. Table 5 also lists the results of Pearson's and Spearman's tests for the positive correlation between the velocity dispersion and SFR surface density. Some of the galaxies exhibited a positive correlation, except for NGC 628, NGC 1365, and the bar region of NGC 3627; the positive correlations are highly significant except for the Circinus galaxy and the arm region of NGC 1365, both of which have only 11 data points.

Finally, Fig. 6 illustrates how the SFR surface density depends on the velocity dispersion and ${ }^{12} \mathrm{CO}(J=1-0)$ luminosity surface density. We fitted a linear relation for the log values of SFR surface density as a function of the velocity dispersion and ${ }^{12} \mathrm{CO}(J=1-0)$ luminosity surface density using the following formula:

$\log ($ SFR surface density $)=$

$$
\alpha_{2} \log \left(L_{12} \mathrm{CO}(J=1-0) \text { surface density }\right)+
$$$$
\beta_{3} \log \text { (velocity dispersion) }+C_{4} \text {. }
$$

The results of these fittings are presented in Table 6. Most of the galaxies exhibit negative $\beta 3$ (velocity dispersion terms) except for NGC 4568 and the Circinus galaxy. We note that all the data points are confined in a narrow hyper surface.

\section{Discussion}

The power-law indices of NGC 628, NGC 1808, and the Circinus galaxy are close to the values of the traditional KS law for the relation between the ${ }^{12} \mathrm{CO}(J=1-0)$ luminosity surface density and SFR surface density (Fig. 3). However, the power-law index identified for NGC 4568 and the arm region of NGC 3627 is lower than for the typical KS law. Additionally, both bar and arm regions of NGC 1365 exhibit a higher power-law index value than the typical KS law. We did not find a correlation between the ${ }^{12} \mathrm{CO}(J=1-0)$ luminosity surface densities and SFR surface densities for the bar region of NGC 3627 and the arm region of NGC 1365. Bigiel et al. (2008) argued that various star-formation laws can exist for different environments and that the KS law indices could range from 0.75 to 2, which roughly relate to different surface number densities of giant molecular clouds (GMCs) within the observed area.

The origin causing the variation from the KS law index is still unclear. Casasola et al. (2015) find that the KS law power index tends to be greater than 1 with spatial resolutions $>100 \mathrm{pc}$ and less than 1 with spatial resolutions $<100 \mathrm{pc}$. Bigiel et al. (2008) found a KS law power index of 1.0 when they selected 
T.-M. Wang and C.-Y. Hwang: Influence of velocity dispersions on star-formation activities in galaxies

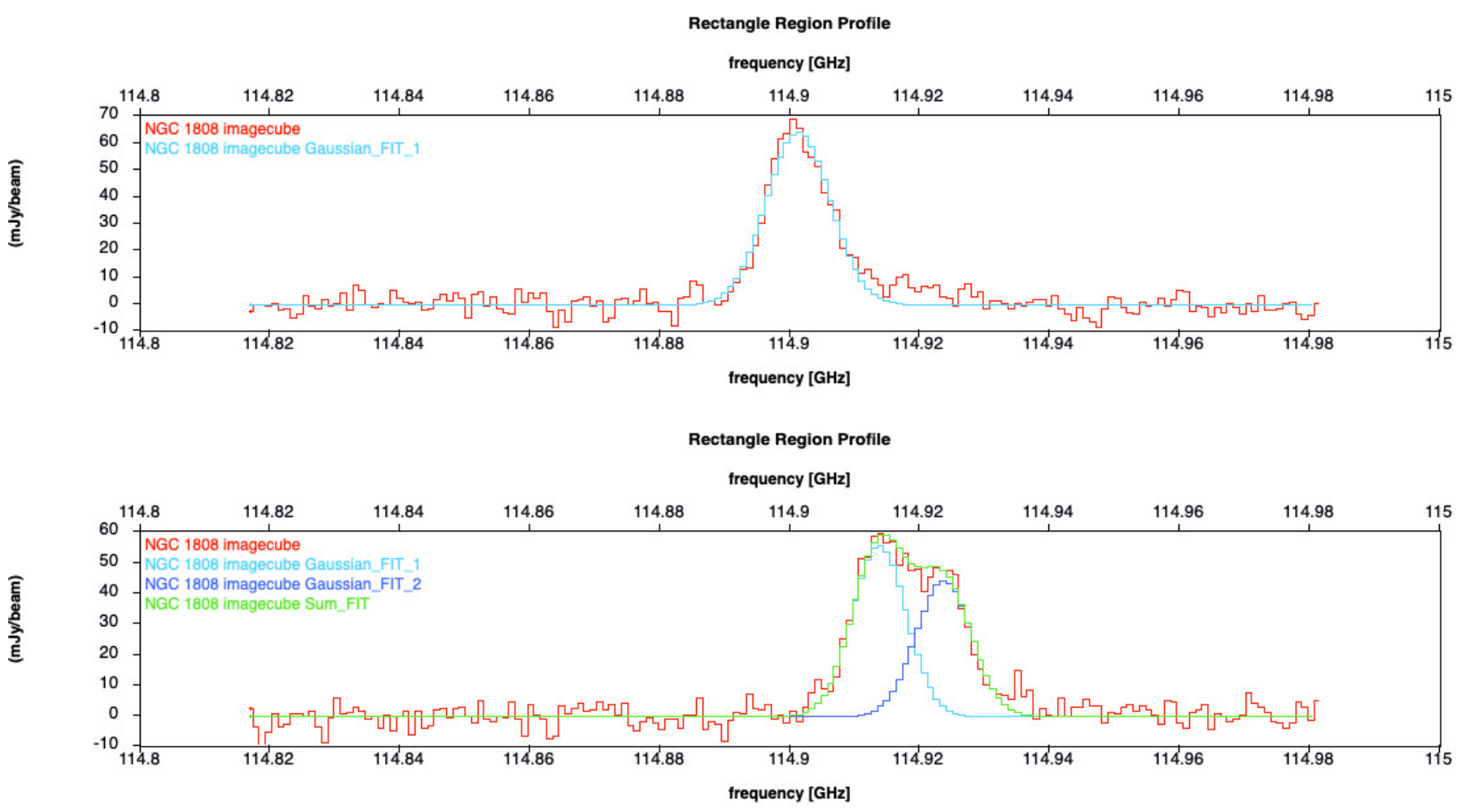

Fig. 2. Using NGC 1808 as an example of how we get velocity dispersion. Top: spectra of the moment 0 map box number 4 and the Gaussian fit of the spectra. Bottom: spectra of the moment 0 map box number 1 and the Gaussian fit of the spectra.

Table 3. Correlation between the ${ }^{12} \mathrm{CO}(J=1-0)$ luminosity surface density and SFR surface density.

\begin{tabular}{lccccccc}
\hline \hline $\begin{array}{l}\text { Galaxy } \\
(1)\end{array}$ & $\begin{array}{c}N \\
(2)\end{array}$ & $\begin{array}{c}r \\
(3)\end{array}$ & $\begin{array}{c}p_{r} \\
(4)\end{array}$ & $\begin{array}{c}\rho \\
(5)\end{array}$ & $\begin{array}{c}p_{\rho} \\
(6)\end{array}$ & $\begin{array}{c}\alpha_{1} \\
(7)\end{array}$ & $\begin{array}{c}C_{1} \\
(8)\end{array}$ \\
\hline NGC628 & 97 & 0.49 & 0.00 & 0.33 & 0.00 & $1.21 \pm 0.13$ & $2.25 \pm 0.34$ \\
NGC1808 & 20 & 0.89 & 0.00 & 0.87 & 0.00 & $1.56 \pm 0.22$ & $2.14 \pm 0.38$ \\
NGC4568 & 48 & 0.82 & 0.00 & 0.84 & 0.00 & $0.75 \pm 0.07$ & $1.15 \pm 0.15$ \\
Circinus galaxy & 11 & 0.85 & 0.00 & 0.91 & 0.00 & $1.80 \pm 0.20$ & $2.55 \pm 0.33$ \\
NGC3627 arm & 35 & 0.80 & 0.00 & 0.81 & 0.00 & $0.83 \pm 0.07$ & $1.10 \pm 0.13$ \\
NGC3627 bar & 9 & -0.58 & 0.10 & -0.68 & 0.04 & $-0.28 \pm 0.14$ & $-1.44 \pm 0.31$ \\
NGC1365 bar & 26 & 0.73 & 0.00 & 0.84 & 0.00 & $2.91 \pm 0.39$ & $3.19 \pm 0.51$ \\
NGC1365 arm & 11 & 0.21 & 0.54 & 0.36 & 0.28 & $6.10 \pm 5.68$ & $12.37 \pm 12.52$ \\
\hline
\end{tabular}

Notes. This table shows (1) name, (2) number of data points for fitting, (3) the Pearson correlation coefficient, (4) the probability of the Pearson correlation, (5) the Spearman rank correlation, (6) the probability of the Spearman rank correlation, (7) the fitting power law index in Eq. (6), (8) the fitting constant in Eq. (6).

Table 4. Correlation between the velocity dispersion and ${ }^{12} \mathrm{CO}(J=1-0)$ luminosity surface density.

\begin{tabular}{lccccccc}
\hline \hline $\begin{array}{l}\text { Galaxy } \\
(1)\end{array}$ & $N$ & $r$ & $p_{r}$ & $\rho$ & $p_{\rho}$ & $\beta_{1}$ & $\begin{array}{c}C_{2} \\
(2)\end{array}$ \\
\hline NGC628 & 97 & 0.13 & 0.20 & 0.04 & 0.73 & $2.92 \pm 0.50$ & $-4.81 \pm 0.37$ \\
NGC1808 & 20 & 0.83 & 0.00 & 0.80 & 0.00 & $3.28 \pm 0.49$ & $-6.16 \pm 0.71$ \\
NGC4568 & 48 & 0.51 & 0.00 & 0.62 & 0.00 & $1.80 \pm 0.20$ & $-4.15 \pm 0.20$ \\
Circinus galaxy & 11 & 0.13 & 0.70 & 0.01 & 0.98 & $1.68 \pm 0.90$ & $-3.86 \pm 1.32$ \\
NGC3627 arm & 35 & 0.68 & 0.00 & 0.60 & 0.00 & $1.85 \pm 0.23$ & $-4.13 \pm 0.26$ \\
NGC3627 bar & 9 & 0.26 & 0.50 & 0.35 & 0.36 & $3.44 \pm 2.46$ & $-6.40 \pm 3.13$ \\
NGC1365 bar & 26 & 0.17 & 0.41 & 0.02 & 0.93 & $4.12 \pm 1.24$ & $-7.27 \pm 1.82$ \\
NGC1365 arm & 11 & 0.15 & 0.66 & 0.13 & 0.70 & $0.38 \pm 0.33$ & $-2.68 \pm 0.42$ \\
\hline
\end{tabular}

Notes. This table shows (1) name, (2) number of data points for fitting, (3) the Pearson correlation coefficient, (4) the probability of the Pearson correlation, (5) the Spearman rank correlation, (6) the probability of the Spearman rank correlation, (7) the fitting power law index in Eq. (7), (8) the fitting constant in Eq. (5). 
Table 5. Correlation between the velocity dispersion and SFR surface density.

\begin{tabular}{lccccccc}
\hline \hline $\begin{array}{l}\text { Galaxy } \\
(1)\end{array}$ & $\begin{array}{c}N \\
(2)\end{array}$ & $\begin{array}{c}r \\
(3)\end{array}$ & $\begin{array}{c}p_{r} \\
(4)\end{array}$ & $\begin{array}{c}\rho \\
(5)\end{array}$ & $\begin{array}{c}p_{\rho} \\
(6)\end{array}$ & $\begin{array}{c}\beta_{2} \\
(7)\end{array}$ & $\begin{array}{c}C_{3} \\
(8)\end{array}$ \\
\hline NGC628 & 97 & 0.00 & 1.00 & -0.12 & 0.25 & $7.78 \pm 3.90$ & $-6.78 \pm 2.94$ \\
NGC1808 & 20 & 0.72 & 0.00 & 0.65 & 0.00 & $5.23 \pm 1.13$ & $-7.63 \pm 1.63$ \\
NGC4568 & 48 & 0.46 & 0.00 & 0.52 & 0.00 & $1.60 \pm 0.25$ & $-2.22 \pm 0.26$ \\
Circinus galaxy & 11 & 0.17 & 0.62 & -0.05 & 0.89 & $9.03 \pm 13.52$ & $-13.10 \pm 19.82$ \\
NGC3627 arm & 35 & 0.51 & 0.00 & 0.48 & 0.00 & $3.11 \pm 1.26$ & $-4.09 \pm 1.44$ \\
NGC3627 bar & 9 & -0.17 & 0.66 & -0.08 & 0.83 & $-5.63 \pm 11.59$ & $6.33 \pm 14.75$ \\
NGC1365 bar & 26 & 0.02 & 0.92 & -0.22 & 0.30 & $10.47 \pm 4.95$ & $-15.50 \pm 7.24$ \\
NGC1365 arm & 11 & -0.04 & 0.91 & 0.12 & 0.73 & $3.89 \pm 2.95$ & $-5.95 \pm 3.71$ \\
\hline
\end{tabular}

Notes. This table shows (1) name, (2) number of data points for fitting, (3) the Pearson correlation coefficient, (4) the probability of the Pearson correlation, (5) the Spearman rank correlation, (6) the probability of the Spearman rank correlation, (7) the fitting power law index in Eq. (8), (8) the fitting constant in Eq. (7).

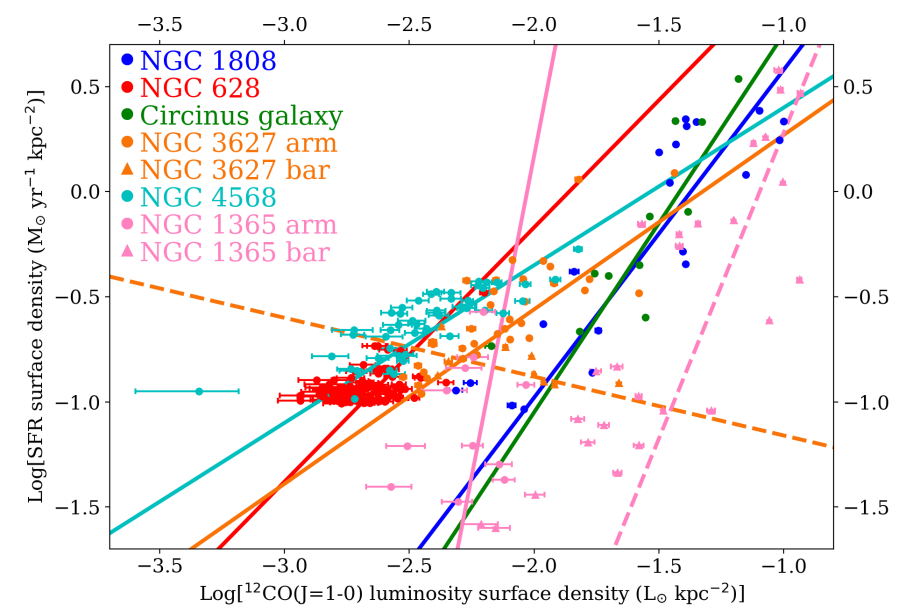

Fig. 3. Relation of the ${ }^{12} \mathrm{CO}(J=1-0)$ luminosity surface density and SFR surface density. Different galaxies are marked in different colors. We fit a linear relation for the log values between these data points. The solid pink circles and the solid pink line of NGC 1365 show the data and the fitting result from the arm region, respectively, and the solid pink triangles and the dashed pink line display the data and the fitting result from the bar region, respectively. The solid orange circles and the solid orange line of NGC 3627 show the data and the fitting result from the arm region, respectively, and the solid orange triangles and the dashed orange line display the data and the fitting result from the bar region, respectively.

a star-forming region with a resolution of $750 \mathrm{pc}$ and $\Sigma_{\mathrm{H} 2}=$ 3-50 $M_{\odot} \mathrm{pc}^{-2}$. They expected at least a few GMCs in their beam, and stressed these 1.0 KS law power indices may reflect the prediction that the properties of GMCs are universal in nearby spiral galaxies. Liu et al. (2011) find that the KS law power indices could increase from 1.2 to 1.8 when the spatial scales decrease from $1.0 \mathrm{kpc}$ to $250 \mathrm{pc}$ for M51a, and from 1.2 to 1.4 when the spatial scales decrease from 700 pc to 250 pc for NGC 3521 .

Casasola et al. (2015) have also studied the molecular gas KS law within the inner kpc of the AGN in the nucleus of NGC 3627. They discovered the KS law power indices in $\sim 1.1$ for spatial resolution of 36,82 , and $200 \mathrm{pc}$ in the central region of NGC 3627 . We note that the result presented in our studying are among the bar and arm regions, which are different from the central region investigated by Casasola et al. (2015).

Our heterogeneous KS law results may reflect that the $200 \mathrm{pc}$ region we used to estimate the star-formation law may contain various physical environments in the GMC for different galaxies.

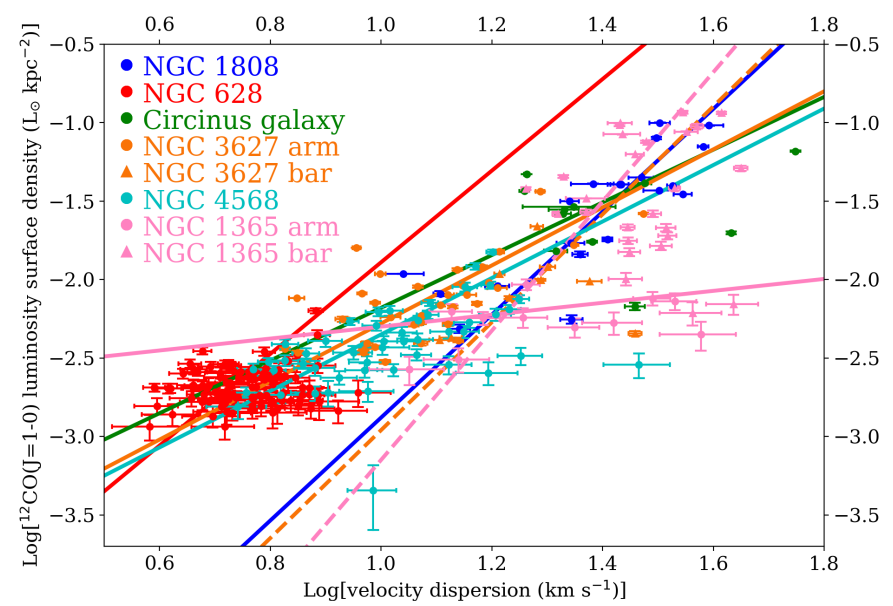

Fig. 4. Relation of the velocity dispersion and ${ }^{12} \mathrm{CO}(J=1-0)$ luminosity surface density. Different galaxies are marked in different colors as shown in the legend. The orthogonal distance regression fittings for different galaxies are displayed as straight lines in different colors. The solid pink circles and the solid pink line of NGC 1365 show the data and the fitting result from the arm region, respectively, and the solid pink triangles and the dashed pink line display the data and the fitting result from the bar region, respectively. The solid orange circles and the solid orange line of NGC 3627 show the data and the fitting result from the arm region, respectively, and the solid orange triangles and the dashed orange line display the data and the fitting result from the bar region, respectively.

For example, Onodera et al. (2010) discovered that the KS law on Local Group spiral galaxy M33 is well correlated in $\sim 1 \mathrm{kpc}$ scale but breaks down in the GMC scale; the breakdown could be attributed to the different evolutionary stages of GMCs. On the other hand, the high turbulent pressure might reduce the starforming activities in hundred-parsec scales of galaxies, leading the KS law power index to decrease. For example, Kruijssen et al. (2014) find that the volume density threshold for star formation increases when there is high turbulent pressure in the central molecular zone (CMZ, i.e., the central $500 \mathrm{pc})$.

We combined our data with the results of typical KS law (Kennicutt 1998) in Fig. 7. To estimate the molecular gas mass surface densities, we used the relation between the $\mathrm{CO}$ integrated line flux density and molecular mass (Bolatto et al. 2013):

$M_{\mathrm{mol}}=1.05 \times 10^{4}\left(\frac{X_{\mathrm{CO}}}{2 \times 10^{20} \frac{\mathrm{cm}^{-2}}{\mathrm{~K} \mathrm{~km} \mathrm{~s}^{-1}}}\right) \frac{S_{\mathrm{CO}} \Delta v D_{\mathrm{L}}^{2}}{(1+z)}$, 


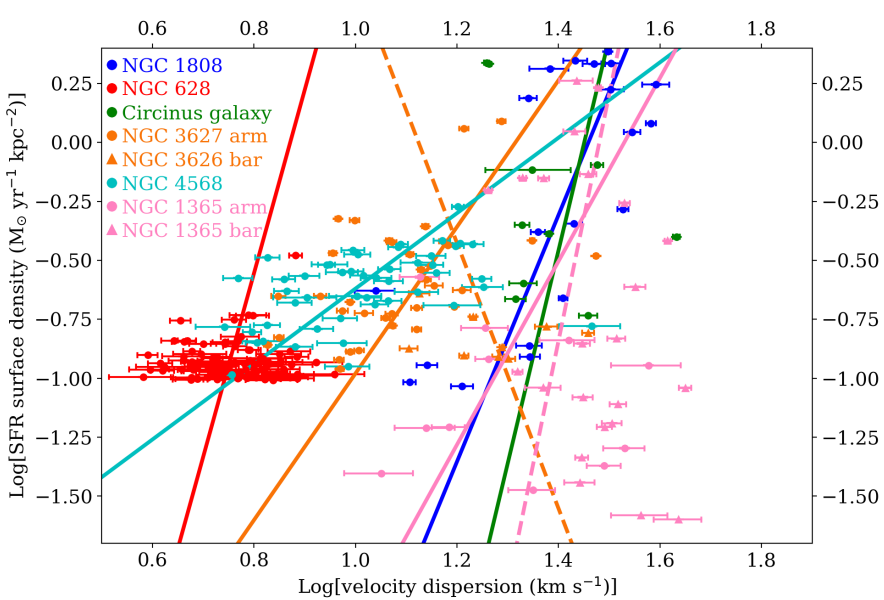

Fig. 5. Relation of the velocity dispersion and SFR surface density. Different galaxies are marked in different colors as shown in the legend. The orthogonal distance regression fittings for different galaxies are displayed as straight lines in different colors. The solid pink circles and the solid pink line of NGC 1365 show the data and the fitting result from the arm region, respectively, and the solid pink triangles and the dashed pink line display the data and the fitting result from the bar region, respectively. The solid orange circles and the solid orange line of NGC 3627 show the data and the fitting result from the arm region, respectively, and the solid orange triangles and the dashed orange line display the data and the fitting result from the bar region, respectively.

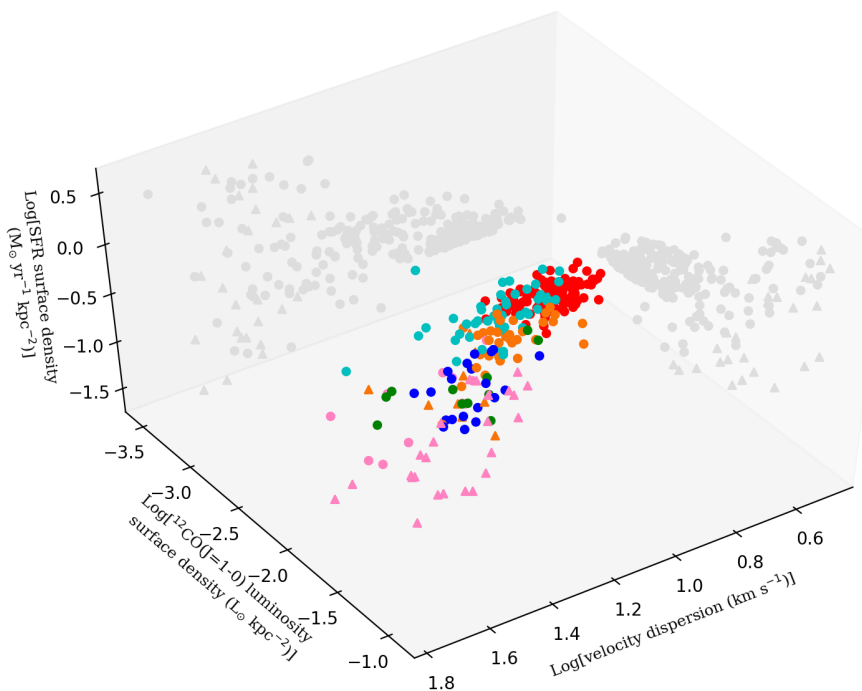

Fig. 6. SFR surface density as a function of the velocity dispersion and ${ }^{12} \mathrm{CO}(J=1-0)$ luminosity surface density. Different galaxies are marked in different colors as shown in Fig. 4. The gray points are the projected data on the SFR surface density-CO luminosity surface density and SFR surface density-velocity dispersion planes.

where $X_{\mathrm{CO}}$ is the CO-to- $\mathrm{H}_{2}$ conversion factor in units of $\mathrm{cm}^{-2}\left(\mathrm{~K} \mathrm{~km} \mathrm{~s}^{-1}\right)^{-1}, S_{\mathrm{CO}} \Delta v$ is the integrated line flux density, $D_{\mathrm{L}}$ is the luminosity distant, and $z$ is the redshift. The value of CO-to- $\mathrm{H}_{2}$ conversion factor $X_{\mathrm{CO}}$ is affected by the physical conditions of molecular gas and could be different in different galaxies. We used the conversion factor derived from previous studies and adopted $X_{\mathrm{CO}}=2.4 \times 10^{20}$ for NGC 628 (Sandstrom et al. 2013), $X_{\mathrm{CO}}=0.8 \times 10^{20}$ for NGC 3627 (Sandstrom et al. $2013), X_{\mathrm{CO}}=1.2 \times 10^{20}$ for NGC 1365 (Tabatabaei et al. 2013), $X_{\mathrm{CO}}=0.4 \times 10^{20}=$ for the Circinus galaxy (Zhang et al. 2014b), and $X_{\mathrm{CO}}=0.8 \times 10^{20}$ for NGC 1808 (Salak et al. 2014). We did not find the CO-to- $\mathrm{H}_{2}$ conversion factor for NGC 4568; we thus adopted the $X_{\mathrm{CO}}=1.8 \times 10^{20}$ for NGC 4568 following Kaneko et al. (2017). We note that most of the conversion factors mentioned above include the contribution of Helium, except for the conversion factor for NGC 1365. We thus multiplied the conversion factor of Tabatabaei et al. (2013) by a factor of 1.36 to include the contribution of Helium.

Our data points are close to the fitting result of the typical KS law. We note that our molecular gas mass surface densities are slightly greater than the gas mass surface densities of the normal galaxies in the traditional KS law because we only selected high $\mathrm{CO}$ intensity regions based on the $\mathrm{CO}$ moment 0 map. Therefore we tended to select regions with higher molecular gas mass than the averaged galaxy gas mass surface density. Our estimated molecular gas surface densities are higher than that of Bigiel et al. $\left(2008 ; \sim 10-10^{2} M_{\odot} \mathrm{pc}^{-2}\right)$ but are consistent with the molecular gas density of Casasola et al. (2015; $\sim 10^{2}-10^{3} M_{\odot} \mathrm{pc}^{-2}$ ). The difference in gas mass surface densities between our result and Bigiel et al. (2008) may reflect the different resolutions used in our study (200 pc) and Bigiel et al. (2008; $750 \mathrm{pc})$. On the other hand, our resolution is similar to the resolution of Casasola et al. (2015), which ranged from 20 to $200 \mathrm{pc}$. Although the surface densities of our galaxies are higher than those of normal galaxies, they are still lower than those of starburst galaxies. Our data points can connect the region between the normal galaxies and starburst galaxies in the KS law fitting (Fig. 7).

Star-formation activities could be affected by AGNs, galaxy mergers, or galactic bars. Previous study has suggested that the interacting galaxies may have higher gas content than normal galaxies and trigger star formation (e.g., Casasola et al. 2004). The AGN activities could enhance or inhibit star-formation activities depending on the stage of AGNs (e.g., Tsai \& Hwang 2015). Moreover, the AGN outflows might also produce a negative influence on star formation as recently discovered for several nearby AGNs with ALMA (e.g., Combes et al. 2013; GarcíaBurillo et al. 2015). These outflows might indeed reduce the main fuel for star formation, the molecular gas. Barred galaxies may have stronger star-formation activities and higher molecular gas concentrations within the central bar region (e.g., Sheth et al. 2005; Wang et al. 2012). We note that NGC 3627 and NGC 4568 are interacting galaxies, and that the high molecular density in these two galaxies might also be affected by the merging process. Furthermore, NGC 1365 and NGC 3627 are barred galaxies, therefore the bar phenomena might also influence the molecular density in these two galaxies. Some of our galaxies might contain very weak AGN activities, such as NGC 1808 and NGC 3627; however, we have minimized the influence of AGN activities by excluding the central regions of galaxies in our study.

We also fitted the KS law power index by using the data points obtained from this work and the normal galaxy parts of Kennicutt (1998). The fitting result gives a KS law power index $N=1.24 \pm 0.04$. This value is close to the traditional KS law power index. However, if we fit the KS law by only using our data points, the fitting result gives an $N=0.94 \pm 0.06$, which is consistent with previous investigation on KS law with a sub-kpc resolution in nearby spiral galaxies (Bigiel et al. 2008).

Positive correlations were observed between the velocity dispersions and ${ }^{12} \mathrm{CO}(J=1-0)$ luminosity surface densities of the studied galaxies (Fig. 4).These results are consistent with those of previous studies; for example, Goto \& Toft (2015) present a weak but significant positive correlation between the $L_{\mathrm{CO}}^{\prime}$ and its Full width at half maximum (FWHM). The observed 
Table 6. SFR surface density as a function of the velocity dispersion and ${ }^{12} \mathrm{CO}(J=1-0)$ luminosity surface density.

\begin{tabular}{lccc}
\hline \hline Galaxy & $\alpha_{2}$ & $\beta_{3}$ & $C_{4}$ \\
\hline NGC628 & $0.32 \pm 0.06$ & $-0.06 \pm 0.09$ & $-0.03 \pm 0.18$ \\
NGC1808 & $1.22 \pm 0.27$ & $-0.12 \pm 0.68$ & $1.87 \pm 1.31$ \\
NGC4568 & $0.47 \pm 0.06$ & $0.07 \pm 0.10$ & $0.46 \pm 0.21$ \\
Circinus galaxy & $1.35 \pm 0.30$ & $0.16 \pm 0.52$ & $1.72 \pm 0.93$ \\
NGC3627 arm & $0.79 \pm 0.14$ & $-0.11 \pm 0.25$ & $1.23 \pm 0.52$ \\
NGC3627 bar & $-0.22 \pm 0.13$ & $-0.02 \pm 0.29$ & $-1.27 \pm 0.51$ \\
NGC1365 bar & $1.53 \pm 0.19$ & $-1.22 \pm 0.73$ & $3.44 \pm 1.12$ \\
NGC1365 arm & $0.73 \pm 0.67$ & $-0.44 \pm 0.60$ & $1.14 \pm 1.99$ \\
\hline All galaxies & $0.84 \pm 0.06$ & $-0.61 \pm 0.11$ & $1.83 \pm 0.24$ \\
All galaxies (exclude bar regions) & $0.80 \pm 0.05$ & $-0.26 \pm 0.09$ & $1.43 \pm 0.21$ \\
\hline
\end{tabular}

Notes. This table shows the fitting results of Eq. (9).

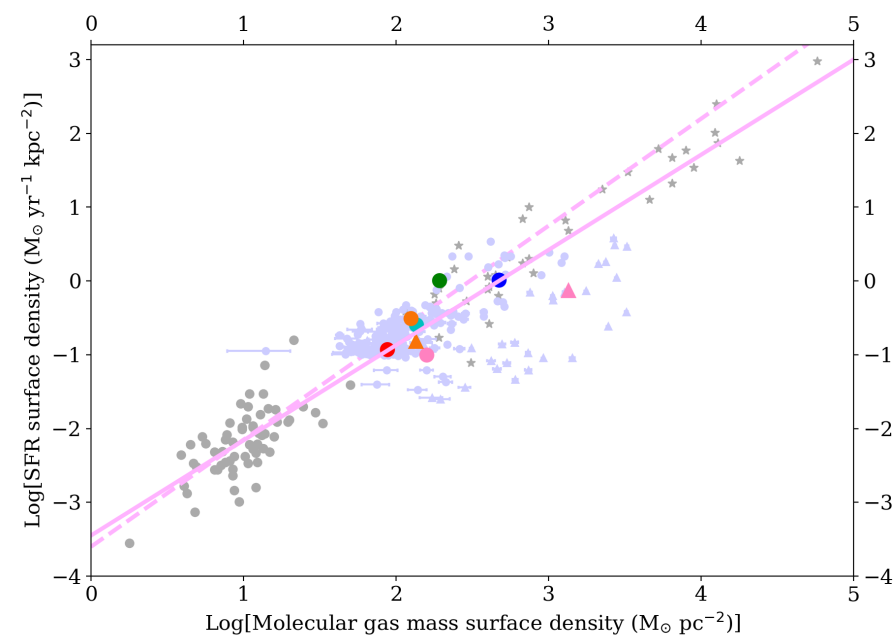

Fig. 7. Relation of the gas mass surface density and the SFR surface density. We combined our data with the results of Kennicutt (1998) The gray points represent the results of Kennicutt Kennicutt (1998). The purple points represent the results for the selected box within our work. The red, magenta, cyan, blue, and orange points represent the average values of the results for each galaxy within our work. The solid pink line shows the fitting result of the data points obtained from this work and normal galaxies obtained from Kennicutt (1998), and the dashed pink line shows the fitting result of typical KS law.

non-significant results for the Circinus galaxy and the arm region of NGC 1365 may have been caused by having too few data points. NGC 628 also has a non-significant positive correlation and shows a lower velocity dispersion compared to other galaxies. However, the velocity dispersion identified in the present results is similar to the $\mathrm{CO}(J=3-2)$ velocity dispersion of $4.1 \pm 2.5 \mathrm{~km} \mathrm{~s}^{-1}$, observed by Wilson et al. (2011) using the James Clerk Maxwell Telescope. This finding suggests that the low velocity dispersion of NGC 628 is real. NGC 628 has the lowest velocity dispersion compared to other galaxies. The velocity dispersion is mainly controlled by the local gravitational potential, which is determined by the distribution of the total baryonic mass, including the stellar mass. We estimated the stellar mass distribution using the Spitzer $3.6 \mu \mathrm{m}$ luminosity (Zhu et al. 2010):

$\log _{10} \frac{M_{*}}{M_{\odot}}=(-0.79 \pm 0.03)+(1.19 \pm 0.01) \times \log _{10} \frac{v L_{\nu}[3.6 \mu m]}{L_{\odot}}$.

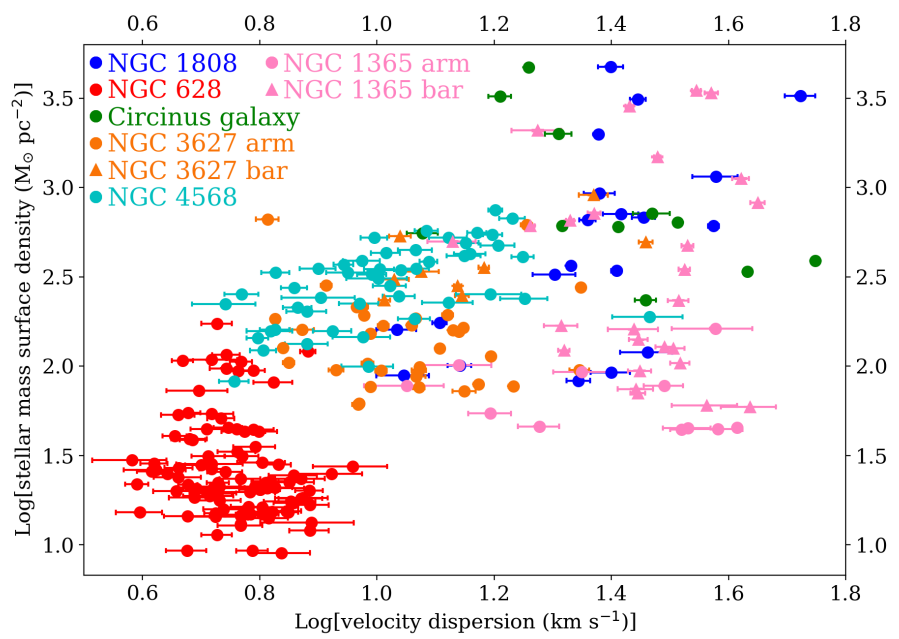

Fig. 8. Relation of the velocity dispersion and stellar mass surface density. Different galaxies are marked in different colors as shown in the legend. The solid pink circles of NGC 1365 show the data from the bar region, and the solid pink triangles display the data from the arm region.

The relations between the velocity dispersion and the stellar mass surface density for all of the studied galaxies are plotted in Fig. 8. We note that NGC 628 exhibits a considerably lower stellar mass surface density than all the other galaxies, even though the molecular gas mass surface density of NGC 628 is only slightly smaller than other typical galaxies, for example, NGC 4568 (i.e., Fig. 4). We used the J-band data from the 2MASS Large Galaxy Atlas to compare the surface brightness of the studied galaxies (Fig. 9). The surface brightness of NGC 628 is the lowest, again indicating that NGC 628 has the lowest stellar mass potential compared with the other galaxies. The results presented above confirm that the velocity dispersion of NGC 628 is real.

The velocity dispersion and SFR surface density of the studied galaxies usually exhibited positive correlations (Fig. 5). These results are close to previously reported results for the global SFR of galaxies, which indicate higher SFRs with larger velocity dispersions (Green et al. 2010). The present results also indicate that star-formation activities were significantly inhibited by the gas motion that we observed. The observed nonsignificance of the positive correlations for the Circinus galaxy and the arm region of NGC 1365 may have been caused by an insufficiency of data points as mentioned before. 
T.-M. Wang and C.-Y. Hwang: Influence of velocity dispersions on star-formation activities in galaxies

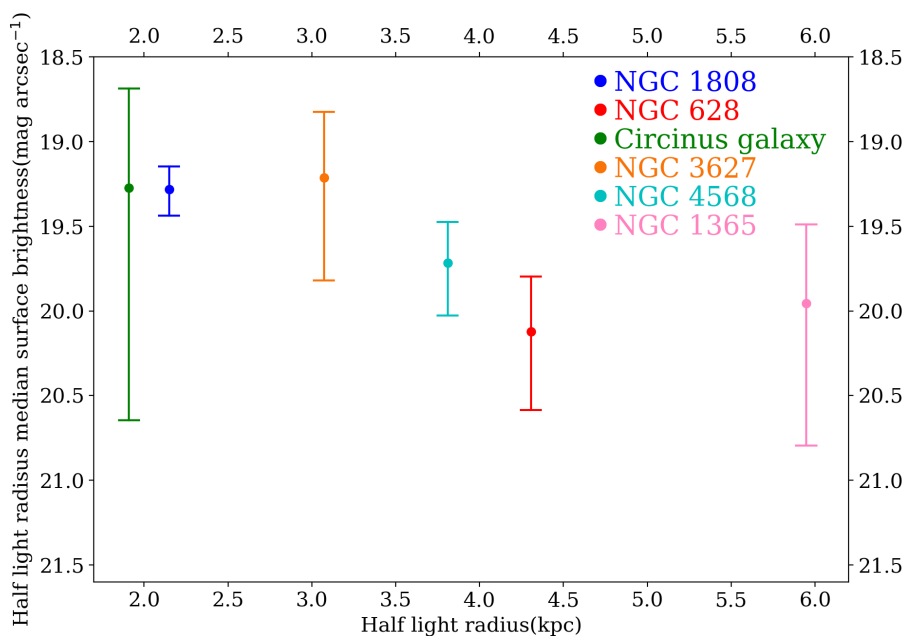

Fig. 9. Relation of the 2MASS $J$-band half light radius and half light radius median surface brightness. Different galaxies are marked in different colors as shown in the legend.

We identified a new fundamental plane among the SFR surface density, velocity dispersion, and ${ }^{12} \mathrm{CO}(J=1-0)$ luminosity surface density for the studied galaxies (Fig. 6). This finding indicates that the results for the star-formation law were influenced by gas surface density and gas motion. In general, the SFR surface density correlated with the gas surface density but anticorrelated with the random motion of the molecular clouds. By fitting our data, we obtained the following modified starformation law:

$$
\begin{aligned}
& \log \text { ( SFR surface density })= \\
& \quad\left(0.84_{ \pm 0.06}\right) \log \left(L_{12} \mathrm{CO}(J=1-0) \text { surface density }\right)+ \\
& \quad\left(-0.61_{ \pm 0.11}\right) \log (\text { velocity dispersion })+\left(1.83_{ \pm 0.24}\right)
\end{aligned}
$$

The anticorreltion between the random motion and the SFR surface density on the fundamental plane is in contrast to those of the positive correlations between the velocity dispersion and SFR surface density (Table 5). We excluded the data points from the bar regions then fitted the equation again. The new fitting results show $\alpha_{2}=0.80 \pm 0.05, \beta_{3}=-0.26 \pm 0.09$, and $\mathrm{C}_{4}=1.43 \pm 0.21$. This result suggests that the gas motion within the bar regions has a more negative influence on star formation than that of the arm regions. It suggests that the star-formation mechanisms might be slightly different in the bar regions and the arm regions. We marked different ${ }^{12} \mathrm{CO}(J=1-0)$ luminosity surface density intervals with different colors on the relation between the velocity dispersion and SFR surface density as shown in Fig. 10. In Fig. 10 we show that the SFR abilities are regulated by the random motion of the molecular clouds within a fixed molecular gas mass density interval. Krumholz \& McKee (2005) found that the SFR surface density is related to the gas surface density with a power law exponent of 0.89 , and to the Toomre parameter with a power law exponent of -1.32 ; their result indicated that the random gas motion could produce negative influence on star formation activities.

Boxes 37, 38, and 39 of NGC 3627 have high ${ }^{12} \mathrm{CO}(J=1-0)$ luminosity surface densities and SFR surface densities compared to other star-formation regions within NGC 3627. We note that these boxes are located at the boundary around the southern arm and the bar of NGC 3627. Previous studies show that a molecular cloud complex with high gas surface density and high SFR density exists within the boundary around the arm and the bar of our

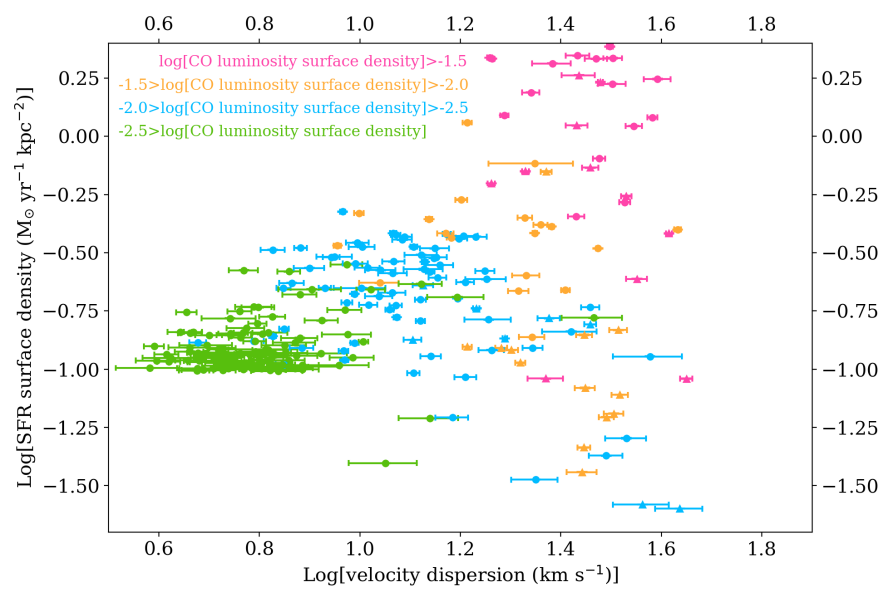

Fig. 10. Relation of the velocity dispersion and SFR surface density. Different molecular gas mass densities are marked in different colors as shown in the legend.

Milky Way; Nguyen Luong et al. (2011) show that the molecular cloud complex W47 has increasing SFR and high clump mass $\left(\sim 8 \times 10^{5} M_{\odot}\right)$, in contrast to normal molecular clouds.

The rotation of the galaxy may contribute shear motion within our $200 \mathrm{pc}$ size scale. It is hard to tell whether the local motion or the shear motion contributes more to the velocity dispersion we observed within the $200 \mathrm{pc}$ size scale from the PV diagram. We conducted a test with the following assumptions: 1. the random gas motions are Gaussian distribution within a box; 2. the standard deviation of the random motion is $10 \mathrm{~km} \mathrm{~s}^{-1}$, which is close to our results of velocity dispersion; 3 . different layers within the box have different shear velocities. If the maximum shear velocity within the box is $10.8 \mathrm{~km} \mathrm{~s}^{-1} 1$, the standard deviation of the gas motion will become $10.6 \mathrm{~km} \mathrm{~s}^{-1}$. If the maximum shear velocity within the box is $30.6 \mathrm{~km} \mathrm{~s}^{-1}$, the standard deviation of the gas motion will become $13.9 \mathrm{~km} \mathrm{~s}^{-1}$. Most of the rotation curves of spiral galaxies do not change more than $30 \mathrm{~km} \mathrm{~s}^{-1}$ within $200 \mathrm{pc}$; therefore, our result indicates that the shear velocity would not contribute significantly to our velocity dispersion results, even if the value of shear velocity is three times greater than the value of the standard deviation of the random motion.

Relatively large scatters are evident in our data distributions. The box size of $200 \times 200 \mathrm{pc}^{2}$ employed in this study may have been slightly larger than the sizes of the real star-forming regions. Some of our boxes may have contained only a small fraction of the corresponding star-formation regions, which in turn may have led to underestimations of the SFR surface densities and ${ }^{12} \mathrm{CO}(J=1-0)$ luminosity surface densities used in comparisons of the velocity dispersion. This could lead to some scatter in the correlations of our data.

For some of the studied galaxies, no total power data were available, which might have caused a missing flux problem. The uv coverage of interferometric observations cannot be complete in general and would cause some large-scale fluxes to go undetected when converting the visibility to images. This problem could be fixed by combining a single-dish total power observation with the interferometric visibility. Among the galaxies without total power data, a total flux of approximately $300 \mathrm{Jy} \mathrm{km} \mathrm{s}^{-1}$ was calculated for NGC 4568, compared with a previously reported total flux of approximately $1500 \mathrm{Jy} \mathrm{km} \mathrm{s}^{-1}$ (Chung et al. 2009). For NGC 3627, a total flux of approximately $440 \mathrm{Jy} \mathrm{km} \mathrm{s}^{-1}$ within the inner $50^{\prime \prime} \times 50^{\prime \prime}$ area was calculated, 
compared with a total flux of approximately $1097 \mathrm{Jy} \mathrm{km} \mathrm{s}^{-1}$ within the inner $50^{\prime \prime} \times 50^{\prime \prime}$ area (Casasola et al. 2011). For NGC 1365 , a total flux of approximately $1454.69 \mathrm{Jy} \mathrm{km} \mathrm{s}^{-1}$ within the inner $45^{\prime \prime} \times 45^{\prime \prime}$ was calculated, compared with the previously reported total flux of approximately $1451.19 \mathrm{Jy} \mathrm{km} \mathrm{s}^{-1}$ (Curran et al. 2000). These differences suggest that a missing flux problem affected the findings for NGC 4568 and NGC 3627. However, the calculations of the $\mathrm{CO}$ luminosity surface density were based on small-scale regions, which may have been less affected by the missing flux that distributed over a much larger scale. Additionally, if the missing flux were taken into consideration, the corrected data would still distribute similarly in the fundamental plane, which is presented in a log-log-log plot.

\section{Summary and conclusions}

We study how the random velocity of molecular gas might influence the star-formation activities in six nearby galaxies. We obtained the ${ }^{12} \mathrm{CO}(J=1-0)$ luminosity surface density and velocity dispersion in $\mathrm{CO}$ emission regions using the archival data of ALMA. We estimated the SFR surface density using the 3.6 and $8 \mu \mathrm{m}$ data of the Spitzer Space telescope. The fitting result of the KS law power index when using our 200 pc box size data points is about $0.94 \pm 0.06$, which is consistent with previous studies investigating sub-kpc KS law in nearby galaxies. We find that the velocity dispersion is correlated with the ${ }^{12} \mathrm{CO}(J=1-0)$ luminosity surface density, which is positively correlated with SFR surface density. However, we discovered that star-forming regions with higher random gas motion have lower star-formation activity. We identified a new fundamental plane among the SFR surface density, velocity dispersion, and ${ }^{12} \mathrm{CO}(J=1-0)$ luminosity surface density, and fitted a new equation for the fundamental plane with a modified KS law. We conclude that the random motion of gas could produce negative influence on star formation.

Acknowledgements. This work was partially supported by the Ministry of Science and Technology (MOST) of Taiwan through grant MOST-1072119-M-008-009-MY3. This paper makes use of the following ALMA data: ADS/JAO.ALMA\#2013.1.00532.S, ADS/JAO.ALMA\#2012.1.01004.S, ADS/JAO.ALMA\#2012.1.00759.S， ADS/JAO.ALMA\#2013.1.00247.S， ADS/ JAO.ALMA\#2015.1.01538.S, ADS/JAO.ALMA\#2015.1.01135.S. ALMA is a partnership of ESO (representing its member states), NSF (USA) and NINS (Japan), together with NRC (Canada), MOST and ASIAA (Taiwan), and KASI (Republic of Korea), in cooperation with the Republic of Chile. The Joint ALMA Observatory is operated by ESO, AUI/NRAO and NAOJ. Also this paper is based [in part] on observations made with the Spitzer Space Telescope, which is operated by the Jet Propulsion Laboratory, California Institute of Technology under a contract with NASA. Support for this work was provided by NASA through an award issued by JPL/Caltech. This publication makes use of data products from the Two Micron All Sky Survey, which is a joint project of the University of Massachusetts and the Infrared Processing and Analysis Center/California Institute of Technology, funded by the National Aeronautics and Space Administration and the National Science Foundation.

\section{References}

Bigiel, F., Leroy, A., Walter, F., et al. 2008, AJ, 136, 2846 Boggs, P. T., \& Rogers, J. E. 1990, Contemp. Math., 112, 186 Bolatto, A. D., Wolfire, M., \& Leroy, A. K. 2013, ARA\&A, 51, 207
Busch, G., Eckart, A., Valencia-S, M., et al. 2017, A\&A, 598, A55 Casasola, V., Bettoni, D., \& Galletta, G. 2004, A\&A, 422, 941 Casasola, V., Hunt, L. K., Combes, F., et al. 2011, A\&A, 527, A92 Casasola, V., Hunt, L., Combes, F., et al. 2015, A\&A, 577, A135 Casasola, V., Cassarà, L. P., Bianchi, S., et al. 2017, A\&A, 605, A18 Chemin, L., Cayatte, V., Balkowski, C., et al. 2003, A\&A, 405, 89 Chung, E. J., Rhee, M.-H., Kim, H., et al. 2009, ApJS, 184, 199 Combes, F., García-Burillo, S., Casasola, V., et al. 2013, A\&A, 558, A124 Cortese, L. 2012, A\&A, 543, A132

Curran, S. J., Aalto, S., \& Booth, R. S. 2000, A\&AS, 141, 193

Eales, S. A., Smith, M. W. L., Wilson, C. D., et al. 2010, A\&A, 518, L62 Elmouttie, M., Koribalski, B., Gordon, S., et al. 1998, MNRAS, 297, 49 Federrath, C. 2013, MNRAS, 436, 1245

Federrath, C., Rathborne, J. M., Longmore, S. N., et al. 2016, ApJ, 832, 143 Ford, G. P., Gear, W. K., Smith, M. W. L., et al. 2013, ApJ, 769, 55 García-Burillo, S., Combes, F., Usero, A., et al. 2015, A\&A, 580, A35 Goto, T., \& Toft, S. 2015, A\&A, 579, A17 Green, A. W., Glazebrook, K., McGregor, P. J., et al. 2010, Nature, 467, 684 Hao, C.-N., Kennicutt, R. C., Johnson, B. D., et al. 2011, ApJ, 741, 124 Helou, G., Roussel, H., Appleton, P., et al. 2004, ApJS, 154, 253 Higuchi, K., Machida, M. N., \& Susa, H. 2018, MNRAS, 475, 3331 Ho, L. C., Filippenko, A. V., \& Sargent, W. L. W. 1997, ApJS, 112, 315 Hull, C. L. H., Mocz, P., Burkhart, B., et al. 2017, ApJ, 842, L9 Kaneko, H., Kuno, N., Iono, D., et al. 2017, PASJ, 69, 66 Kennicutt, R. C. 1998, ApJ, 498, 541

Kennicutt, R. C., Calzetti, D., Walter, F., et al. 2007, ApJ, 671, 333

Kirk, H., Johnstone, D., \& Basu, S. 2009, ApJ, 699, 1433

Kruijssen, J. M. D., Longmore, S. N., Elmegreen, B. G., et al. 2014, MNRAS, 440, 3370

Krumholz, M. R., \& McKee, C. F. 2005, ApJ, 630, 250

Leitherer, C., Schaerer, D., Goldader, J. D., et al. 1999, ApJS, 123, 3

Leroy, A. K., Walter, F., Brinks, E., et al. 2008, AJ, 136, 2782

Liu, G., Koda, J., Calzetti, D., et al. 2011, ApJ, 735, 63

McKee, C. F., \& Ostriker, E. C. 2007, ARA\&A, 45, 565

Miura, R. E., Kohno, K., Tosaki, T., et al. 2014, ApJ, 788, 167

Momose, R., Koda, J., Kennicutt, R. C., et al. 2013, ApJ, 772, L13

Moorwood, A. F. M., Lutz, D., Oliva, E., et al. 1996, A\&A, 315, L109

Nguyen Luong, Q., Motte, F., Schuller, F., et al. 2011, A\&A, 529, A41

Norman, C., \& Silk, J. 1980, ApJ, 238, 158

Ondrechen, M. P., \& van der Hulst, J. M. 1989, ApJ, 342, 29

Onodera, S., Kuno, N., Tosaki, T., et al. 2010, ApJ, 722, L127

Orkisz, J. H., Pety, J., Gerin, M., et al. 2017, A\&A, 599, A99

Ott, J., \& Kern, J. 2016, CASA Synthesis \& Single Dish Reduction Reference Manual \& Cookbook, National Radio Astronomy Observatory

Padoan, P., Federrath, C., Chabrier, G., et al. 2014, Protostars and Planets VI, eds. H. Beuther, R. S. Klessen, C. P. Dullemond, \& T. Henning, (Tucson: University of Arizona Press), 77

Pereira-Santaella, M., Colina, L., García-Burillo, S., et al. 2016, A\&A, 587, A44 Peretz, U., \& Behar, E. 2018, MNRAS, 481, 3563

Ramos Almeida, C., Levenson, N. A., Rodríguez Espinosa, J. M., et al. 2009, ApJ, 702, 1127

Reif, K., Mebold, U., Goss, W. M., et al. 1982, A\&AS, 50, 451

Salak, D., Nakai, N., \& Kitamoto, S. 2014, PASJ, 66, 96

Sandstrom, K. M., Leroy, A. K., Walter, F., et al. 2013, ApJ, 777, 5

Schmidt, M. 1959, ApJ, 129, 243

Sheth, K., Vogel, S. N., Regan, M. W., et al. 2005, ApJ, 632, 217

Shostak, G. S., \& van der Kruit, P. C. 1984, A\&A, 132, 20

Shu, F. H., Adams, F. C., \& Lizano, S. 1987, ARA\&A, 25, 23

Tabatabaei, F. S., Weiß, A., Combes, F., et al. 2013, A\&A, 555, A128

Tsai, M., \& Hwang, C.-Y. 2015, AJ, 150, 43

Venturi, G., Nardini, E., Marconi, A., et al. 2018, A\&A, 619, A74

Véron-Cetty, M.-P., \& Véron, P. 2010, A\&A, 518, A10

Vollmer, B., Soida, M., Beck, R., et al. 2013, A\&A, 553, A116

Wang, J., Kauffmann, G., Overzier, R., et al. 2012, MNRAS, 423, 3486

Wilson, C. D., Warren, B. E., Irwin, J., et al. 2011, MNRAS, 410, 1409

Wu, H., Cao, C., Hao, C.-N., et al. 2005, ApJ, 632, L79

Zhang, Q., Qiu, K., Girart, J. M., et al. 2014a, ApJ, 792, 116

Zhang, Z.-Y., Henkel, C., Gao, Y., et al. 2014b, A\&A, 568, A122

Zhu, Y.-N., Wu, H., Li, H.-N., et al. 2010, Res. Astron. Astrophys., 10, 329 
T.-M. Wang and C.-Y. Hwang: Influence of velocity dispersions on star-formation activities in galaxies

\section{Appendix A: Moment 0 maps for the selected galaxies}
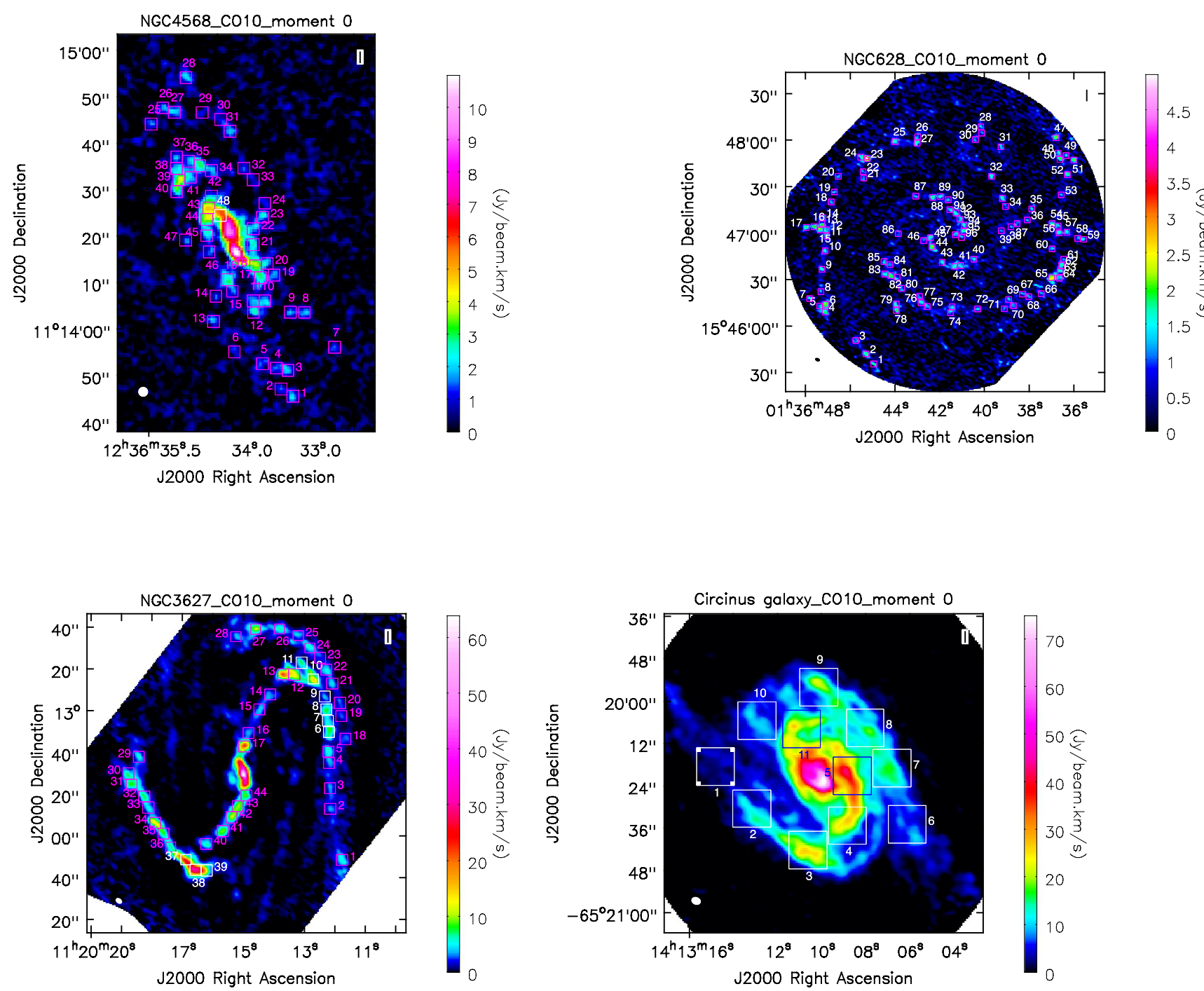

Fig. A.1. ${ }^{12} \mathrm{CO}(J=1-0)$ moment 0 maps and boxes selected for NGC 4568 (top left), NGC 628 (top right), NGC 3627 (bottom left), and the Circinus galaxy (bottom right).
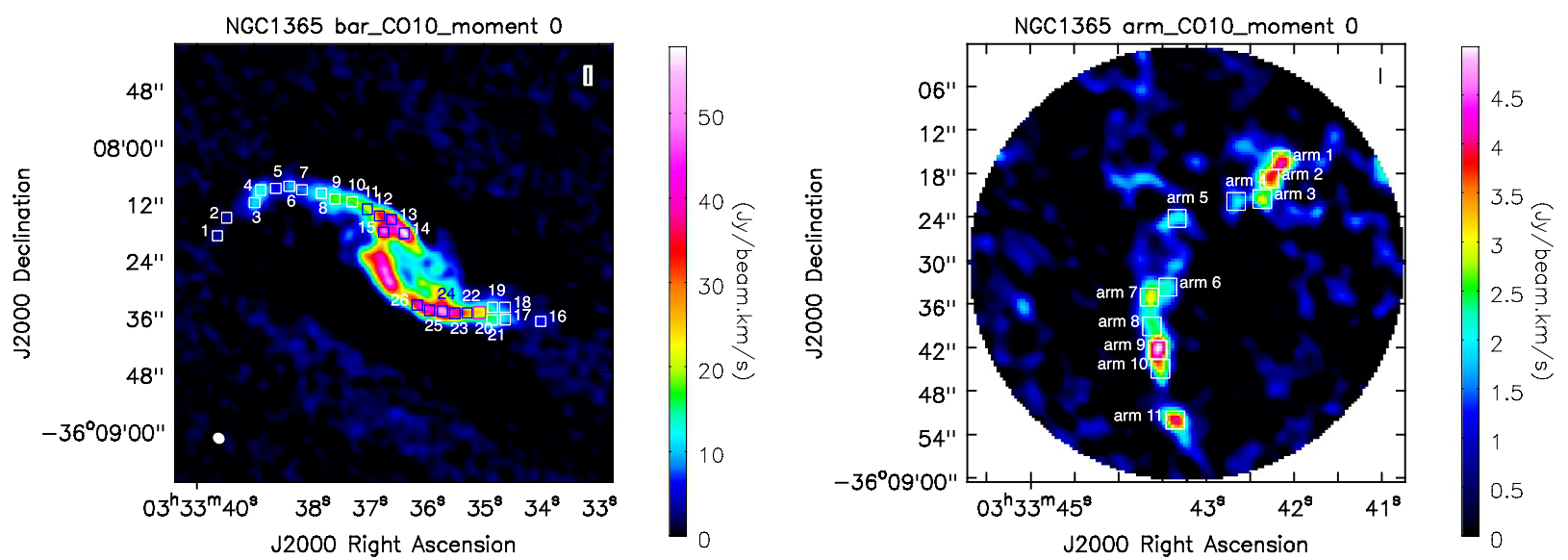

Fig. A.2. ${ }^{12} \mathrm{CO}(J=1-0)$ moment 0 maps and boxes selected for NGC1365. We separated NGC 1365 into a bar (left) and an arm (right) region for different cleaning. 